\title{
Multimodal probe for optical coherence tomography epidetection and micron-scale indentation
}

\author{
L. Bartolini*, F. Feroldi, J. J. A. Weda, M. Slaman, J. F. de Boer and D. Iannuzzi \\ Department of Physics and Astronomy \\ Vrije Universiteit Amsterdam and LaserLab Amsterdam \\ de Boelelaan 1081, 1081HV Amsterdam, The Netherlands \\ *l.bartolini@vu.nl
}

Received 10 July 2017

Accepted 15 September 2017

Published 17 October 2017

\begin{abstract}
We present a multimodal ferrule-top sensor designed to perform the integrated epidetection of Optical Coherence Tomography (OCT) depth-profiles and micron-scale indentation by all-optical detection. By scanning a sample under the probe, we can obtain structural cross-section images and identify a region-of-interest in a nonhomogeneous sample. Then, with the same probe and setup, we can immediately target that area with a series of spherical-indentation measurements, in which the applied load is known with a $\mu \mathrm{N}$ precision, the indentation depth with sub- $\mu \mathrm{m}$ precision and a maximum contact radius of $100 \mu \mathrm{m}$. Thanks to the visualization of the internal structure of the sample, we can gain a better insight into the observed mechanical behavior. The ability to impart a small, confined load, and perform OCT $A$-scans at the same time, could lead to an alternative, high transverse resolution, Optical Coherence Elastography (OCE) sensor.
\end{abstract}

Keywords: Optomechanical; microindentation; optical coherence tomography; indentation; multimodal sensor; epidetection.

\section{Introduction}

The importance of mechanical measurements is undisputedly recognized in several research areas. In the field of biomedical research, for instance, quantitative assessments of the viscoelastic properties of cells and tissues allow scientists to investigate the role of mechanics in the physiology of living systems. ${ }^{1-3}$ This kind of studies may eventually lead to major breakthroughs in tissue engineering and in the detection of life-threatening diseases. ${ }^{4,5}$

The local mechanical properties of biological materials are typically assessed via Atomic Force Microscopy (AFM $)^{6-9}$ by means of so-called nanoindentation techniques. A tip, mounted at the end of a micro-machined cantilever spring, is pushed into the sample with a calibrated stroke. By looking at how the deflection of the cantilever evolves over

*Corresponding author.

This is an Open Access article published by World Scientific Publishing Company. It is distributed under the terms of the Creative Commons Attribution 4.0 (CC-BY) License. Further distribution of this work is permitted, provided the original work is properly cited. 


\section{Bartolini et al.}

time, one can infer the mechanical properties of the material underneath the indented point. ${ }^{10,11}$

Indentation techniques, however, suffer from a main limitation: they measure the collective behavior of the volume of material underneath the contact area: when researchers observe spatial variations of mechanical properties, they cannot tell whether those differences arise from the presence of heterogeneous structures in the sensed volume, or if that volume is homogeneous and simply possesses different mechanical properties.

This ambiguity is particularly detrimental when indentation is used as a diagnostic tool to discern healthy tissues from diseased ones. Biological samples, indeed, typically present a high degree of multiscale structures which affect their macroscopic behavior. $^{12-14}$

A visualization of the inner structures of the sample would allow for a better interpretation of mechanical measurements and for a disambiguation between the influence of internal structures and intrinsic mechanical differences. A noteworthy technique for this purpose is Optical Coherence Tomography (OCT), which offers a field-of-view and a resolution relevant to the scales of nano- and micro-indentation.

In 1998, Schmitt published the seminal paper of Optical Coherence Elastography (OCE), ${ }^{15}$ in which he combined OCT imaging to assess strain in the material and mechanical loading to impart a known compressive stress, and eventually obtained a visualization of the mechanical properties of a sample called elastogram. There are now multiple approaches to the creation of elastograms by OCE, differing in the type and spatial extent of the loading mechanism, or in its temporal characteristics. ${ }^{16-20}$ To aim at applications in life sciences, researchers put considerable effort in increasing the resolution of elastograms, targeting the finer structures of tissues that may be early indicators of disease. Recent OCE works obtained micro-scale resolution $^{21-24}$ elastograms, improving the OCT signal processing to extract more accurate strain measurements and reducing the contact area to apply a more localized mechanical stimulus; effort in miniaturization resulted in the needle-based OCE for in vivo and in situ characterization. ${ }^{25-27}$

Over the last few years, our group has pioneered a new indentation technique that has been tailored for applications in life science research. ${ }^{28-30}$ The technique relies on a ferrule-top probe, which is obtained by assembling a millimeter-size cantilever, equipped with $\mathrm{a} \approx 100 \mu \mathrm{m}$ diameter sphere on its free hanging end on a small glass block. The block hosts an optical fiber used to measure the deflection of the cantilever and reproduce the indentation protocol used in AFM nano-indentation. This approach has already been used by several groups to assess the mechanical properties of tissues and cells. ${ }^{11,31-38}$ Furthermore, in 2013, we have demonstrated that the ferrule-top probes used for indentation can be modified to host another optical fiber that, connected with an OCT system, enables the user to look at how the subsurface features of a sample deform when the indenter is pushed into the sample. ${ }^{39}$ Those studies have never been brought beyond a first proof-of-concept, whose practical relevance in life sciences was very limited: the use of new fabrication techniques and materials lead to a reduction of the stiffness of the cantilever by three orders of magnitude with respect to those initial studies, enabling measurements on biologicallyrelevant soft materials. Moreover, the old design was imparting the compressive load by means of a small tube glued to the cantilever, which allowed the OCT signal to pass through. Unfortunately, such indenter geometry posed severe limitations to the quantitative analysis of the mechanical data, its sharp edges lead to stress accumulation and could have damaged soft samples and there is no analytical model for such an indenter shape. This issue was crucially overcome as the new, transparent cantilever allows us to substitute the tube with a hemispherical sapphire indenter tip, which at the same time, acts as a half-ball lens, for focusing and epidetection of one-dimensional (1D) OCT depth profiles ( $A$-scans) along the indentation axis.

Our probe can hover over the surface of a thick sample mounted on motorized stages: by driving them in a raster scan under the fixed sensor, we can obtain cross-sections or volumetric OCT images ( $B$ - or $C$-scans), with which they visualize and identify possible subsurface structures. The probe can then target regions-of-interest and perform quantitative micro-indentation measurements in there.

Other designs combining OCT and microindentation have been published, but they are only applicable to thin samples, since indentation and OCT are performed from different sides of sample. ${ }^{40}$ The integration of indentation and OCT on the same sensor allows for an immediate and accurate co-localization of the optical and mechanical 
measurements with notable gains in speed and repeatability. Moreover, the geometry of the indenter makes it possible to test virgin materials (no need for precompression) at very fine scales, keeping the contact radius below $100 \mu \mathrm{m}$.

We show that with this system, we can indeed acquire $B$-scans of a sample that hides complex features underneath a homogenous surface, and that the probe is then capable of distinguishing different mechanical responses when positioned on top of the different subsurface structures.

\section{Experimental Details}

\subsection{Ferrule-top sensor: Design and fabrication}

Figure 1 shows a microscope image of the probe, which was designed to combine ferrule-top indentation with the acquisition of OCT depth profiles in an epidetection scheme. The probe consists of a borosilicate glass ferrule hosting: (i) a cantilever spring, used to apply a calibrated mechanical load, (ii) a half-ball lens, used both as indenting tip and as focusing element for the OCT signal, and (iii) two optical fibers, one used to measure the deflection of the cantilever and the other used to perform OCT.

The fabrication of the probe consists of three steps: preparation of the ferrule, preparation of the cantilever with the lens, and their assembly.

In the first step, a $3 \mathrm{~mm} \times 3 \mathrm{~mm} \times 7 \mathrm{~mm}$ borosilicate glass ferrule is mounted on a diamond wire cutter, which is used to carve a $3 \mathrm{~mm} \times 500 \mu \mathrm{m} \times$

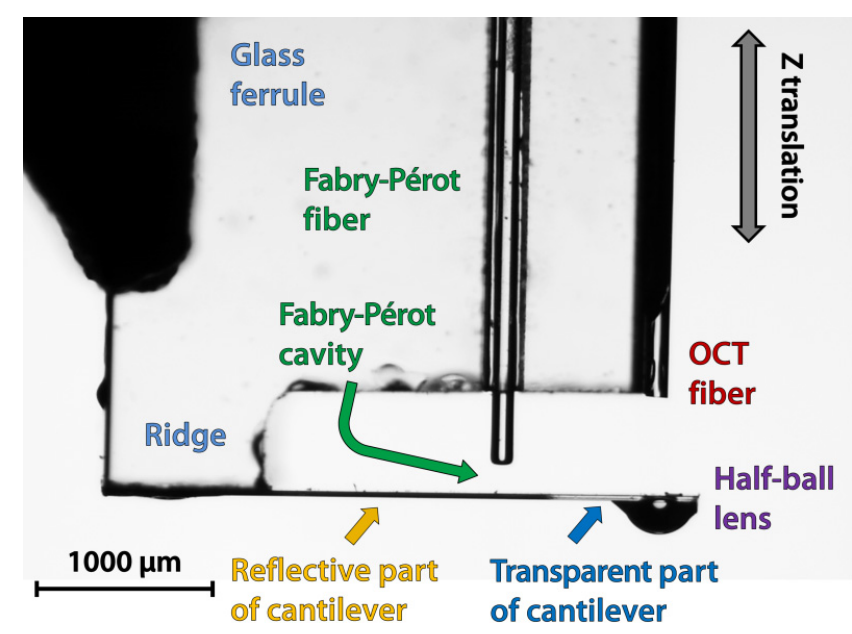

Fig. 1. Microscope side view of the ferrule-top sensor combining indentation and OCT capabilities.
$500 \mu \mathrm{m}$ ridge out of the small facet of the ferrule and a deep groove on its side [Fig. 2(a)].

In the second step [Fig. 2(b)], a borosilicate glass ribbon (Vitrocom Inc.), with a rectangular section of $30 \mu \mathrm{m} \times 300 \mu \mathrm{m}$, is made reflective for most of its length by sputtering a $5 \mathrm{~nm}$ thick chromium adhesion layer followed by a $100 \mathrm{~nm}$ thick gold film. The remaining length of the ribbon, around $500 \mu \mathrm{m}$, is left transparent. There, a sapphire half-ball lens (Edmund Optics, $200 \mu \mathrm{m}$ radius) is glued on the opposite face of the coating.

In the third step [Fig. 2(c)], the ribbon is glued to the ridge of the ferrule. A single mode optical fiber (Corning SMF28) is then cleaved and mounted in the side groove, pointing at the reflective part of the cantilever. Another single mode optical fiber (Corning SMF28), cleaved at an angle of $8^{\circ}$ to suppress back reflections, is glued on the long face of the ferrule, aligned with the lens at the free hanging end of the cantilever.

\subsection{Setup}

The first optical fiber mounted in the side groove and aligned with the reflective part of the cantilever is connected to a commercial interferometer (OP1550, Optics11) to measure, via Fabry-Pérot interferometry, the deflection of the cantilever.

The other optical fiber is connected via a circulator (CIR-1310-50-APC, Thorlabs Inc., Newton, NJ, USA) to the sample arm of a custom swept-sourcebased OCT system that has been described in the previous reports. ${ }^{41,42}$ Light from a $1310 \mathrm{~nm}$ sweptsource laser (A-line rate $=50 \mathrm{kHz}$, optical bandwidth $=90 \mathrm{~nm}$, Axsun Inc., Billerica, MA, USA) is split into a sample arm and a reference arm, set in a MachZehnder modality. About $12 \mathrm{~mW}$ of sample arm light reaches the sensor through the second port of a circulator, which in turn receives the backscattered light collected by the ball lens. The light from the circulator's third port interferes with the reference arm light and the so-called DC terms are rejected through balanced detection (PDB430C, Thorlabs Inc., Newton, NJ, USA), yielding a sensitivity of $112 \mathrm{~dB}$. The acquisition of a $B$-scan (2000 $A$-lines/ $B$-scan) starts when the position of the stage reaches a set location: the stage controller then sends an acquisition-trigger to the high-speed digitizer (ATS9350, Alazar Inc., Pointe Claire, Canada).

The data are processed as follows: the average $k$-space spectrum is subtracted from each individual 
(a)

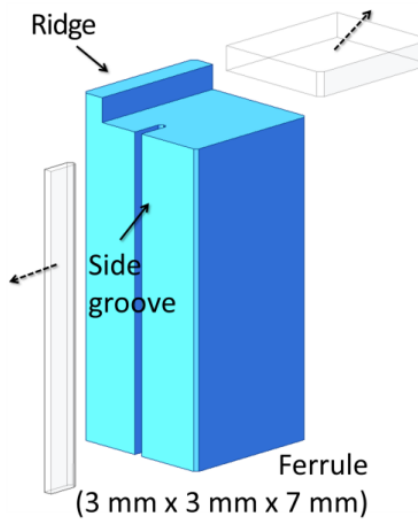

(b)

\section{Cantilever}

(length $3 \mathrm{~mm}$, section $300 \mu \mathrm{m} \times 30 \mu \mathrm{m}$ )

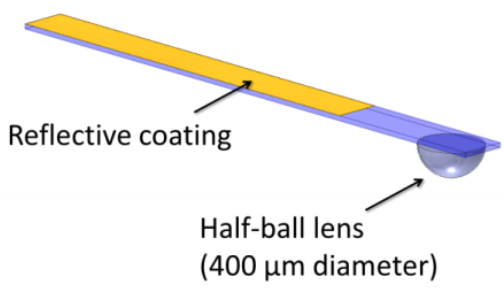

(c)

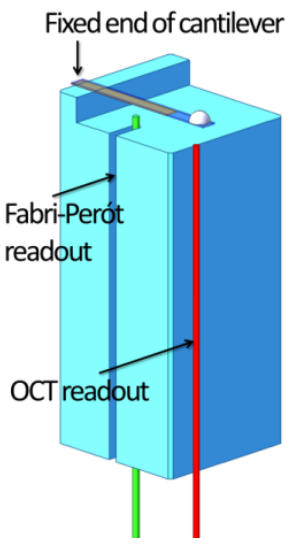

Fig. 2. OCE Ferrule-top fabrication steps: The ferrule is showed upside-down to ease the visualization. (a) preparation of the ferrule (micromachining of the ridge and of the side groove); (b) preparation of the cantilever; and (c) assembly of the two readout fibers and of the cantilever.

spectrum to remove DC noise and fixed patterns in the image. Then, a cosine window and a chromatic dispersion correction curve are applied before performing a Fourier transform (fft, Mathworks Inc., Natick, MA, USA). Finally, the resulting absolute values are converted in $\mathrm{dB}$ scale, producing an $A$-line.

The ferrule-top probe is mounted on a threedimensional (3D) printed rigid arm, as shown in Fig. 3. The arm is fixed to a long-travel piezoelectric device (PI-602.3SL, $300 \mu \mathrm{m}$ range, Physik Instrumente $\mathrm{GmbH}$ ), which in turn is fixed on a coarse $Z$-axis manipulator with a travel of $12.5 \mathrm{~mm}$.

The sample is placed on top of two orthogonal linear stages (M664.164, Physik Instrumente $\mathrm{GmbH}$, driven by $\mathrm{C} 867$ controllers from the same company) that allow for $X-Y$ positioning with an accuracy of less than $1 \mu \mathrm{m}$.

\subsection{Experimental procedure}

To acquire an OCT image, the tip of the sensor is initially brought approximately $800 \mu \mathrm{m}$ above the

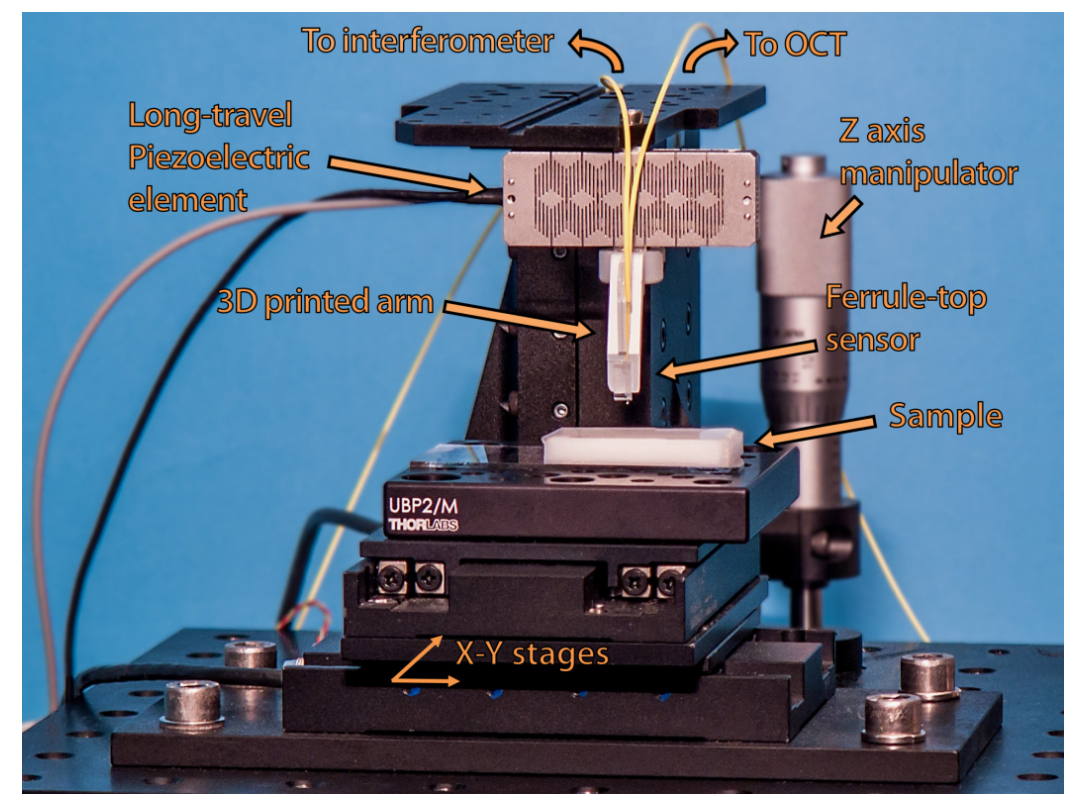

Fig. 3. Overview of the setup: OCT imaging is obtained by moving the sample with the $X-Y$ stages while the sensor hovers at approximately $800 \mu \mathrm{m}$ above the sample. Indentation measurements make use of the interferometric readout and are driven by the piezoelectric element. 
surface of the sample. Using the $X-Y$ stages, the sample is then moved back and forth to allow for $B$-scans and, if required, $C$-scans. The maximum speed for the fast-scan axis is $75 \mathrm{~mm} / \mathrm{s}$. In order to guarantee a regime of constant speed, the motion includes a run-up of $2 \mathrm{~mm}$, after which a hardware trigger from the $X-Y$ stage controller initiates the acquisition of the $B$-scan. Maximum repeatability of the $B$-scans is guaranteed by unidirectional scanning. The axial resolution of the OCT signal depends on its light source, and is around $10 \mu \mathrm{m}$ in PDMS, whereas the lateral resolution (roughly given by the spot size, determined by the geometry of the optical components of the probe) is estimated by Zemax OpticStudio simulations to be around $30 \mu \mathrm{m}$.

Before the indentation experiment, we bring the sensor close to the surface with the $Z$-axis manipulator and extend the piezoelectric device until the tip gets in contact with the surface. Then, the actual mechanical measurement begins: as the tip is pushed into the sample, we record the extension of the piezoelectric translator (via its internal straingauge), the deflection of the cantilever (via the interferometer), and the indentation depth (calculated as difference between the piezoelectric extension and the cantilever deflection). Using a software feedback loop running at $500 \mathrm{~Hz}$, we impose a temporal profile to the indentation depth by adjusting the position of the piezoelectric element. Eventually, we calculate the force required to reach a predefined depth set-point by multiplying the measured deflection of the cantilever by the associated spring constant, previously obtained via a standard calibration technique. ${ }^{43}$

\subsection{Sample preparation}

To demonstrate the working principle of this ferrule-top sensor, we created a sample that, while avoiding the ethical and technical issues of real biological tissues, shares with them several meaningful properties. Such tissue-mimicking phantom was made out of a bi-component crosslinked elastomer polydimethylsiloxane (PDMS, Sylgard 184, Dow Corning) and presents the following characteristics:

- Layers: the sample is composed of two layers.

- Structure: a step defect changes the thickness of the top layer.

- Mechanical contrast: the top layer is stiffer than the bottom one.
- Optical contrast: the bottom layer scatters more than the top one.

The preparation of the sample borrows from a technique used in soft lithography, ${ }^{44}$ in which a positive mask defines the structure imprinted on the bottom surface of a casted polymer. We start by gluing a cover-slip (thickness of $100 \mu \mathrm{m}$ ) to a microscope slide and spray it with a thin layer of Teflon (DuPont, dry-film lubricant) in order to prevent adhesion later on. We place the microscope slide at the bottom of a mold and cast the prepolymer of PDMS inside it. After curing, we peel the polymer off the mask and out of the mold and put it upside-down in the mold, where it is covered with another PDMS layer. Following this procedure, we are able to obtain a sample with a flat surface and a nonhomogeneous subsurface structure. To create mechanical contrast, the bottom layer is made with a ratio of 40:1 elastomer to curing agent, whereas the top layer is tuned to be stiffer by mixing the components in a ratio of 10:1 (higher fraction of curing agent results in more crosslinks and higher stiffness). Furthermore, by substituting different fractions of the elastomer with a special solution (made with $\mathrm{TiO}_{2}$ particles suspended in $\mathrm{OH}-$ terminated silicone oil), we can obtain different scattering coefficients in the two layers, resulting in optical contrast for the OCT image. As expected, the finished sample appears homogeneous at visual inspection (see Fig. 3), yet it is expected to present an internal structure and two layers with different mechanical and optical properties.

\section{Results and Discussion}

To demonstrate the working principle of the probe, we surveyed the sample by performing $B$-scans in different locations to identify the position of the underlying step structure. Then, we included that area-of-interest in a $C$-scan of $3 \times 3 \mathrm{~mm}$ and eventually performed mechanical indentation along a $B$-scan.

In the OCT image, the structure of the sample is clearly recognizable, as shown in the $B$-scan in Fig. 4. Backreflections from the sensor are notable at fixed vertical positions in the upper part of the image, but they are not intense enough to jeopardize image quality. Such backreflections are unavoidable in this type of sensor, as they arise from the mismatch of the indexes of refraction at the four 


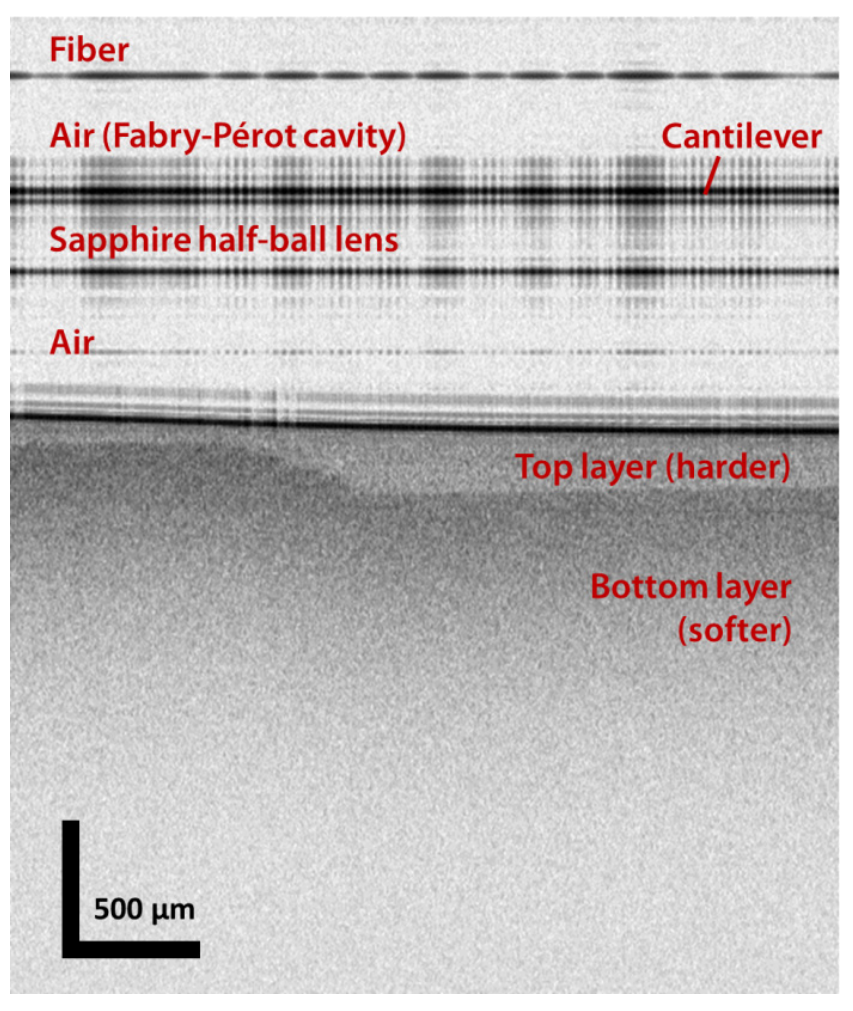

Fig. 4. $B$-scan acquired with the sensor hovering on top of the sample. The total horizontal length is $3 \mathrm{~mm}$, the vertical length is a $5 \mathrm{~mm}$ optical-path-difference, and it has been rescaled to represent the true (physical) size of the PDMS sample. The material of each layer has been labeled, the horizontal lines in the upper part are the backreflections happening at the interfaces of the components of the sensor.

interfaces of the sensor: starting from the top, we identify the fiber-to-air, the air-to-cantilever, the cantilever-to-sapphire-lens and the sapphire-lens-toair interfaces. Measuring in a liquid environment would mitigate the mismatches and the intensity of those backreflections. However, the hydrodynamic forces on the cantilever would then impose an upper limit on the scanning velocity. In air, on the contrary, we can drive the stage at full speed and obtain a $B$-scan in $40 \mathrm{~ms}$ and a $C$-scan in less than $3 \mathrm{~min}$.

In Fig. 4, the contrast between the two layers is due to their different scattering coefficient, tuned to be lower in the top one. It is possible to see a smearing of the defect, resulting in a smoothened transition between the region with a thin top layer and the region with a thicker one. The upper surface is smooth but not perfectly horizontal due to imperfections in the fabrication procedure.

After imaging, we performed mechanical measurements across the defect. Specifically, we measured the load required to reach an indentation

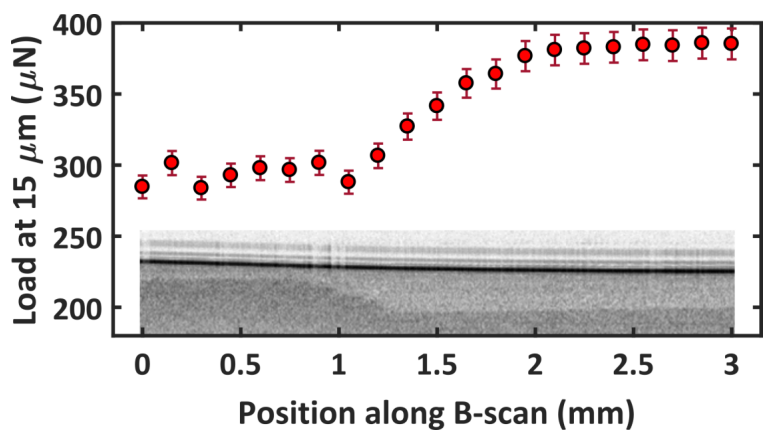

Fig. 5. Load applied to reach $15 \mu \mathrm{m}$ indentation depth, superimposed on the $B$-scan along which the measurements took place. The error bars are due to the error on the spring constant of the cantilever. The 21 measurements are $150 \mu \mathrm{m}$ apart, and they cover the $3 \mathrm{~mm}$ length of the $B$-scan. Load is calculated by multiplying the deflection of the cantilever by its spring constant, quantities which are respectively measured by Fabri-Perot interferometry and by a calibration procedure.

depth of $15 \mu \mathrm{m}$, and repeated that every $150 \mu \mathrm{m}$ along the whole $3 \mathrm{~mm}$ length of the $B$-scan. The results are shown in Fig. 5. In the first approximation, the observed mechanical behavior depends on all the material included in a radius of around 10 times the indentation depth, in our case, extending to around $150 \mu \mathrm{m}$ : this is the depth until which mechanical properties are "sensed". In the region where the harder layer comprises a smaller fraction of such volume, the load to reach $15 \mu \mathrm{m}$ depth is therefore lower than the region in which the top and harder layer is thicker (see Fig. 5). The calibration procedure, from which we obtained a stiffness of the cantilever of $21.3 \pm 0.6 \mathrm{~N} / \mathrm{m}$, is the predominant source of error on the load (see error bars in Fig. 5).

In these measurements, our ability to recognize two areas and ascribe the different observed mechanical behavior to the respective subsurface structures is a direct consequence of the way our sample was created. In fact, the mechanically stiffer PDMS layers presents the lesser scattering coefficient: optical contrast and mechanical contrast had been tailored to have an identical spatial distribution. However, in some samples, such as breastcancer, ${ }^{26}$ the sources of mechanical contrast might be different from the sources of scattering-based optical contrast. In those cases, performing independent imaging and mechanical measurements as done in this paper would not to be as insightful as demonstrated in this work. This leaves room for future developments, aimed at a more intimate integration of OCT imaging and mechanical measurements with the implementation of OCE 
techniques. One major hurdle would be the proper quantification of stress within the sample: most current OCE protocols apply the mechanical stimulus over the whole imaging area, and proceed with the analysis in the assumption of a uniform stress distribution inside the material. Our probe does not require precompression, and exerts loads smaller and more confined that any other quasi-static OCE probe. ${ }^{16}$ While this is desirable in many life sciences applications, it would not allow us to assume that the stress is uniform along the axis of indentation for the whole imaging range. The depth-resolved quantification of mechanical properties would therefore require more refined modeling of the propagation of the stress field in the material upon indentation.

\section{Conclusions}

We showed the working principle of a multimodal probe that combines indentation at micro-scale with OCT in an all-optical epidetection scheme. With this sensor, it is possible to serve two purposes: first, one can hover on a structured sample and identify a morphological area-of-interest via OCT, and second, one can perform localized and accurate mechanical measurements in that area-ofinterest. The epidetection scheme offers the added value of being able to image thick samples, paving the way to a novel quantitative approach to optomechanical investigation of nontransparent, thick tissues.

\section{Acknowledgments}

The authors would like to thank M. Marrese for her input and fruitful discussions. The research leading to these results is supported by the Dutch Technology Foundation (STW) under the OMNE program (13183) and has received funding from LASERLABEUROPE under the EC's Seventh Framework Program (Grant agreement No. 284464) and the European Research Council (615170).

\section{References}

1. G. A. Holzapfel, R. W. Ogden, Mechanics of Biological Tissue, Springer-Verlag, Berlin (2006).

2. R. G. Wells, "Tissue mechanics and fibrosis," Biochim. Biophys. Acta, Mol. Basis Dis. 1832, 884-890 (2013).
3. S. C. Cowin, S. B. Doty, Tissue Mechanics, Springer, New York (2007).

4. I. Lokody, "Microenvironment: Tumour-promoting tissue mechanics," Nat. Rev. Cancer 14, 296-297 (2014).

5. M. Lekka, D. Gil, K. Pogoda, J. Dulińska-Litewka, R. Jach, J. Gostek, O. Klymenko, S. PrauznerBechcicki, Z. Stachura et al., "Cancer cell detection in tissue sections using AFM," Arch. Biochem. Biophys. 518, 151-156 (2012).

6. G. Binnig, C. F. Quate, C. Gerber, "Atomic force microscope," Phys. Rev. Lett. 56, 930-933 (1986).

7. M. L. Crichton, X. Chen, H. Huang, M. A. F. Kendall, "Elastic modulus and viscoelastic properties of full thickness skin characterised at micro scales," Biomaterials 34, 2087-2097 (2013).

8. B. Pittenger, "Advances in AFM nanomechanics AFM provides high resolution mapping of various sample properties" (2013).

9. M. L. Oyen, "Nanoindentation of biological and biomimetic materials," Exp. Tech. 37, 73-87 (2013).

10. M. L. Oyen, "Analytical techniques for indentation of viscoelastic materials," Phil. Mag. 86, 5625-5641 (2006).

11. G. Mattei, G. Gruca, N. Rijnveld, A. Ahluwalia, "The nano-epsilon dot method for strain rate viscoelastic characterisation of soft biomaterials by spherical nano-indentation" J. Mech. Behav. Biomed. Mater. 50, 150-159 (2015).

12. C.-L. Guo, N. C. Harris, S. S. Wijeratne, E. W. Frey, C.-H. Kiang, "Multiscale mechanobiology: Mechanics at the molecular, cellular, and tissue levels," Cell Biosci. 3, 25 (2013).

13. P. Egan, R. Sinko, P. R. LeDuc, S. Keten, "The role of mechanics in biological and bio-inspired systems," Nat. Commun. 6, 7418 (2015).

14. J. L. Katz, A. Misra, P. Spencer, Y. Wang, S. Bumrerraj, T. Nomura, S. J. Eppell, M. Tabib-Azar, "Multiscale mechanics of hierarchical structure/ property relationships in calcified tissues and tissue/ material interfaces," Mater. Sci. Eng. C 27, 450-468 (2007).

15. J. Schmitt, "OCT elastography: Imaging microscopic deformation and strain of tissue," Opt. Express 3, 199-211 (1998).

16. J. A. Mulligan, G. R. Untracht, S. N. Chandrasekaran, C. N. Brown, S. G. Adie, "Emerging approaches for high-resolution imaging of tissue biomechanics with optical coherence elastography," IEEE J. Sel. Top. Quantum Electron. 22, 1-20 (2016).

17. K. V. Larin, D. D. Sampson, "Optical coherence elastography - OCT at work in tissue biomechanics [invited]," Biomed. Opt. Express 8, 1172 (2017).

18. S. Wang, K. V. Larin, "Optical coherence elastography for tissue characterization: A review," $J$. Biophoton. 9999, 1-24 (2014). 
19. B. F. Kennedy, K. M. Kennedy, D. D. Sampson, "A review of optical coherence elastography: Fundamentals, techniques and prospects," IEEE J. Sel. Top. Quantum Electron. 20, 272-288 (2014).

20. B. F. Kennedy, P. Wijesinghe, D. D. Sampson, "The emergence of optical elastography in biomedicine," Nat. Photon. 11, 215-221 (2017).

21. B. F. Kennedy, R. A. McLaughlin, K. M. Kennedy, L. Chin, A. Curatolo, A. Tien, B. Latham, C. M. Saunders, D. D. Sampson, "Optical coherence micro-elastography: Mechanical-contrast imaging of tissue microstructure," Biomed. Opt. Express 5, 2113 (2014).

22. K. M. Kennedy, L. Chin, R. A. McLaughlin, B. Latham, C. M. Saunders, D. D. Sampson, B. F. Kennedy, "Quantitative micro-elastography: Imaging of tissue elasticity using compression optical coherence elastography," Sci. Rep. 5, 15538 (2015).

23. L. Chin, B. F. Kennedy, K. M. Kennedy, P. Wijesinghe, G. J. Pinniger, J. R. Terrill, R. A. McLaughlin, D. D. Sampson, "Three-dimensional optical coherence micro-elastography of skeletal muscle tissue," Biomed. Opt. Express 5, 3090-3102 (2014).

24. S. Es'haghian, K. M. Kennedy, P. Gong, Q. Li, L. Chin, P. Wijesinghe, D. D. Sampson, R. A. McLaughlin, B. F. Kennedy, "In vivo volumetric quantitative micro-elastography of human skin," Biomed. Opt. Express 8, 2458 (2017).

25. K. M. Kennedy, B. F. Kennedy, R. A. McLaughlin, D. D. Sampson, "Needle optical coherence elastography for tissue boundary detection," Opt. Lett. 37, 2310 (2012).

26. K. M. Kennedy, R. A. McLaughlin, B. F. Kennedy, A. Tien, B. Latham, C. M. Saunders, D. D. Sampson, "Needle optical coherence elastography for the measurement of microscale mechanical contrast deep within human breast tissues," J. Biomed. Opt. 18, 121510 (2013).

27. Y. Qiu, Y. Wang, Y. Xu, N. Chandra, J. Haorah, B. Hubbi, B. J. Pfister, X. Liu, "Quantitative optical coherence elastography based on fiber-optic probe for in situ measurement of tissue mechanical properties," Biomed. Opt. Express 7, 688-700 (2016).

28. D. Chavan, T. C. van de Watering, G. Gruca, J. H. Rector, K. Heeck, M. Slaman, D. Iannuzzi, "Ferruletop nanoindenter: An optomechanical fiber sensor for nanoindentation," Rev. Sci. Instrum. 83, 115110 (2012).

29. H. van Hoorn, N. A. Kurniawan, G. H. Koenderink, D. Iannuzzi, "Local dynamic mechanical analysis for heterogeneous soft matter using ferrule-top indentation," Soft Matter 12, 3066-3073 (2016).

30. S. V. Beekmans, D. Iannuzzi, "Characterizing tissue stiffness at the tip of a rigid needle using an opto-mechanical force sensor," Biomed. Microdevices 18, 1-8 (2016).

31. E. J. Bos, K. van der Laan, M. N. Helder, M. G. Mullender, D. Iannuzzi, P. P. van Zuijlen, "Noninvasive measurement of ear cartilage elasticity on the cellular level: A new method to provide biomechanical information for tissue engineering," Plast. Reconstr. Sur. Glob. Open 5, e1147 (2017).

32. C. Lavet, P. Ammann, "Osteoarthritis like alteration of cartilage and subchondral bone induced by protein malnutrition is treated by nutritional essential amino acids supplements," Osteoarthr. Cartilage 25, S 293(2017).

33. B. Sarker, R. Singh, T. Zehnder, T. Forgber, C. Alexiou, I. Cicha, R. Detsch, A. R. Boccaccini, "Bioactive and compatible polymers macromolecular interactions in alginate-gelatin hydrogels regulate the behavior of human fibroblasts," J. Bioact. Compat. Polym. 32, 309-324 (2017).

34. P. R. Moshtagh, B. Pouran, N. M. Korthagen, A. A. Zadpoor, H. Weinans, "Guidelines for an optimized indentation protocol for measurement of cartilage stiffness: The effects of spatial variation and indentation parameters," J. Biomech. 49, 3602-3607 (2016).

35. M. Vashaghian, A. M. Ruiz-Zapata, M. H. Kerkhof, B. Zandieh-Doulabi, A. Werner, J. P. Roovers, T. H. Smit, "Toward a new generation of pelvic floor implants with electrospun nanofibrous matrices: A feasibility study," Neurourol. Urodyn. 36, 565-573 (2017).

36. W. E. G. Müller, E. Tolba, H. C. Schröder, S. Wang, G. Glaßer, R. Muñoz-Espí, T. Link, X. Wang, "A new polyphosphate calcium material with morphogenetic activity," Mater. Lett. 148, 163-166 (2015).

37. S. Wang, X. Wang, F. G. Draenert, O. Albert, H. C. Schröder, V. Mailänder, G. Mitov, W. E. G. Müller, "Bioactive and biodegradable silica biomaterial for bone regeneration," Bone 67, 292-304 (2014).

38. M. Neufurth, X. Wang, H. C. Schröder, Q. Feng, B. Diehl-Seifert, T. Ziebart, R. Steffen, S. Wang, W. E. G. Müller, "Engineering a morphogenetically active hydrogel for bioprinting of bioartificial tissue derived from human osteoblast-like SaOS-2 cells," Biomaterials 35, 8810-8819 (2014).

39. D. Chavan, J. Mo, M. de Groot, A. Meijering, J. F. de Boer, D. Iannuzzi, "Collecting optical coherence elastography depth profiles with a micromachined cantilever probe," Opt. Lett. 38, 1476-1478 (2013).

40. Y. Yang, P. O. Bagnaninchi, M. Ahearne, R. K. Wang, K.-K. Liu, "A novel optical coherence tomography-based micro-indentation technique for mechanical characterization of hydrogels," $J$. R. Soc. Interface 4, 1169-1173 (2007). 
41. J. Li, M. de Groot, F. Helderman, J. Mo, J. M. A. Daniels, K. Grünberg, T. G. Sutedja, J. F. de Boer, "High speed miniature motorized endoscopic probe for optical frequency domain imaging," Opt. Express 20, 24132 (2012).

42. J. Li, F. Feroldi, J. de Lange, J. M. A. Daniels, K. Grünberg, J. F. de Boer, "Polarization sensitive optical frequency domain imaging system for endobronchial imaging," Opt. Express 23, 3390 (2015).
43. S. V. Beekmans, D. Iannuzzi, "A metrological approach for the calibration of force transducers with interferometric readout," Surf. Topogr. Metrol. Prop. 3, 1-12 (2015).

44. S. Walia, C. M. Shah, P. Gutruf, H. Nili, D. R. Chowdhury, W. Withayachumnankul, M. Bhaskaran, S. Sriram, "Flexible metasurfaces and metamaterials: A review of materials and fabrication processes at micro- and nano-scales," Appl. Phys. Rev. 2, 011303 (2015). 